\title{
CircRNA-Cdrlas Exerts Anti-Oncogenic Functions in Bladder Cancer by Sponging MicroRNA-135a
}

\author{
Peng Li $i^{a, b} \quad$ Xiao Yang ${ }^{a}$ Wenbo Yuan ${ }^{a}$ Chengdi Yang ${ }^{a, b}$ Xiaolei Zhang ${ }^{a}$ Jie Han ${ }^{a}$ \\ Jingzi Wang ${ }^{a}$ Xiaheng Deng ${ }^{a}$ Haiwei Yang ${ }^{a}$ Pengchao Lia Jun Tao ${ }^{a}$ Qiang Lu ${ }^{a}$ \\ Min $\mathrm{Gu}^{\mathrm{a}}$ \\ aDepartment of Urology, The First Affiliated Hospital of Nanjing Medical University, Nanjing, \\ bDepartment of Urology, Suzhou Du shu hu Public Hospital (Soochow University Multi-Disciplinary \\ Polyclinic), Suzhou, China
}

\author{
Key Words \\ CircRNA • Bladder cancer $\bullet$ MiR-135a $・$ P21 $\bullet$ Cdr1as
}

\begin{abstract}
Background/Aims: CircRNAs regulate gene expression in different malignancies. However, the role of Cdrlas in the tumourigenesis of bladder cancer and its potential mechanisms remain unknown. Methods: qRT-PCR was used to detect Cdrlas and target miRNA expression in bladder cancer tissues and cell lines. Biological functional experiments were performed to detect the effects of Cdrlas on the biological behaviour of bladder cancer cells in vivo and in vitro. Bioinformatic analysis was utilised to predict potential miRNA target sites on Cdr1as. Ago2 RNA binding protein immunoprecipitation assay, RNA antisense purification assay, biotin pull down assay and RNA FISH were performed to detect the interaction between Cdrlas and target miRNAs. Western blot was used to determine the expression level of p21 in bladder cancer cells. Results: Cdrlas was significantly down-regulated in bladder cancer tissues compared with adjacent normal tissues. Overexpression of Cdrlas inhibited the proliferation, invasion and migration of bladder cancer cells in vitro and slowed down tumour growth in vivo. Cdrlas sponged multiple miRNAs in bladder cancer. Moreover, Cdrlas directly bound to miR-135a and inhibited its activity in bladder cancer. Conclusion: Cdr1as is downregulated and sponges multiple miRNAs in bladder cancer. It exerts anti-oncogenic functions by sponging microRNA-135a.
\end{abstract}

(C) 2018 The Author(s) Published by S. Karger AG, Basel

\section{Introduction}

Bladder cancer is a common malignancy worldwide with an estimated 430, 000 new cases diagnosed in 2012 [1]. Approximately 75\% of newly diagnosed bladder cancers are P. Li, X. Yang and W. Yuan contributed equally to this work.

Qiang Lu

and Min Gu 


\section{Cellular Physiology Cell Physiol Biochem 2018;46:1606-1616 and Biochemistry Published online: April 25, $2018 \quad \begin{aligned} & \text { DOI } 1018 \text { The Author(s). Published by S. Karger AG, Basel } \\ & \text { www.karger.com/cpb }\end{aligned}$ Li et al.: CircRNA-Cdr1as Inhibits Bladder Cancer Proliferation by Sponging microRNA- $135 a$}

non-muscle invasive, $70 \%$ of which may undergo tumour relapse and $25 \%$ may progress to muscle-invasive bladder cancer [2]. Given the high incidence, recurrence rate and progression rate of bladder cancer, the five-year survival rate of patients with this disease remains dismal. Therefore, elucidation of the mechanisms contributing to the development, progression and metastasis of bladder cancer is important.

Non-coding RNAs(ncRNAs) were a kind of RNAs with no protein-coding function that are widely expressed in organisms. NcRNAs played important roles in bladder cancer [3]. For example, microRNAs may regulate the proliferation, migration, invasion $[4,5]$ and the chemo-sensitivity [6] of bladder cancer. Long Non-coding RNAs also showed huge functions in bladder cancer, including tumorigenesis, growth [7], apoptosis [8], metastasis [9] and so on. Recently, circRNAs have been found own great influence in cancer.

CircRNAs are a novel type of RNAs characterised by a covalently closed continuous loop with neither $5^{\prime}-3^{\prime}$ polarities nor polyadenylated tails [10]. They are evolutionarily conserved among different species and often exhibit tissue/developmental-stage-specific expression [11]. Growing evidence has indicated that circRNAs play crucial roles in many biological and pathological processes, including cell proliferation, migration and invasion, metastasis, cell cycle progression and carcinogenesis [12-17]. Recent studies have confirmed that circRNAs are potential biomarkers in many cancers, such as hepatocellular carcinoma [18], colon cancer [19] and gastric cancer [20]. However, little is known about their function in bladder cancer.

Cdr1as, also named ciRS-7 or Cdr1NAT, is $1500 \mathrm{nt}$ long and is transcribed in the antisense orientation with respect to the cerebellum degeneration-related antigen 1 (CDR1) gene [21]. Recent studies have shown that Cdr1as can bind to miR-7 and function as miRNA sponges in different diseases. Ectopic Cdr1 as expression in zebrafish impairs midbrain development, which is similar to knocking down miR-7 [11]. In islet cells, Cdr1as regulates insulin transcription and secretion via miR-7 and its targets [22]. Moreover, it serves as an oncogene in hepatocellular carcinoma through targeting miR-7 expression [23]. In the present study, we found that Cdr1as was down-regulated in bladder cancer tissues compared with adjacent normal tissues (ANTs). Overexpression of Cdr1as can inhibit the proliferation and invasion of bladder cancer cells. Mechanistically, Cdr1as can up-regulate p21 expression by sponging miR-135a.

\section{Materials and Methods}

\section{Clinical specimen collection}

Ninety-four pairs of bladder cancer tissues and ANTs were obtained from bladder cancer patients who underwent radical cystectomy between 2013 and 2016 in the Department of Urology, Jiangsu Provincial People's Hospital. This work has been approved by the Research Ethics Committee of our institution, and all patients signed an informed consent. All specimens were immediately frozen and stored in liquid nitrogen until RNA extraction.

\section{Cell culture}

Human bladder cancer cell lines EJ and T24 were obtained from the Type Culture Collection of the Chinese Academy of Sciences (Shanghai, China). All cells were maintained in DMEM medium containing $10 \%$ fetal bovine serum (FBS; Gibco, Australia) and 1\% penicillin-streptomycin and cultured in an incubator with humidified $5 \% \mathrm{CO}_{2}$ at $37^{\circ} \mathrm{C}$.

Transfection

All miRNA mimics utilised for transfection were purchased from GenePharma (Shanghai, China). The Cdr1as overexpression plasmid was provided by Dr. T. Hansen (Aarhus University, Denmark). The Cdr1as overexpression adenovirus was constructed by Hanbio (Shanghai, China). The transfection procedure was performed in accordance with the manufacturer's protocol. The cells were harvested $48 \mathrm{~h}$ after transfection. 


\section{Cellular Physiology Cell Physiol Biochem 2018;46:1606-1616 \begin{tabular}{l|l|l} 
DOI: 10.1159/000489208 & O 2018 The Author(s). Published by S. Karger AG, Basel \\
and Biochemistry & $\begin{array}{l}\text { Published online: April 25, } 2018 \\
\text { www.com/cpb }\end{array}$
\end{tabular} \\ Li et al.: CircRNA-Cdrlas Inhibits Bladder Cancer Proliferation by Sponging microRNA- \\ $135 a$}

\section{RNA extraction and $q R T-P C R$}

Total RNAs including miRNAs were extracted from tissues and transfected cells using TRIzol reagent (Invitrogen, USA) in accordance with the manufacturer's protocol. Extracted RNA was prepared using an All-in-One ${ }^{T M}$ miRNA qRT-PCR Detection Kit (GeneCopoeia, FulenGen, China) in accordance with the manufacturer's instructions. cDNA was synthesised using HiScript II (Vazyme, China). Real-time qRT-PCR for circRNA and miRNA was performed on an AB7300 thermo-recycler (Applied Biosystems, USA). The primers used were synthesised by Sangon Biotech (Shanghai, China), and their sequences were as follows: Cdr1as (forward: 5'-AGA CCT TGA GAT TAT TGG AAG ACT TGA-3'; reverse: 5'-TAC CCA GTC TTC CAT CAA CTG GCT - $3^{\prime}$ ), $\beta$-actin (forward: $5^{\prime}$-AGC GAG CAT CCC CCA AAG TT-3'; reverse: $5^{\prime}$-GGG CAC GAA GGC TCA TCA TT-3'), miR-7-5p (forward: 5'-UGG AAG ACU AGU GAU UUU GUU GU-3'), miR-135a-5p (forward: $5^{\prime}$-UAU GGC UUU UUA UUC CUA UGU GA-3'), miR-1246 (forward: 5'-AAU GGA UUU UUG GAG CAG G-3'), miR-1290 (forward: $5^{\prime}$-UGG AUU UUU GGA UCA GGG A-3'), universal adaptor reverse primer ( $5^{\prime}$-GGC CAA CCG CGA GAA GAT GTT TTT TTT T-3') and U6 (forward: 5'-CTC GCT TCG GCA GCA CA-3'; reverse: 5'-AAC GCT TCA CGA ATT TGC GT-3'). $\beta$-actin and U6 RNAs were used as internal standard controls for mRNA and miRNA detection, respectively. Each experiment was replicated three times, and data were analysed using the $2^{-\Delta \Delta C T}$ method.

\section{Protein extraction and Western blot}

Total protein was extracted using RIPA lysis buffer (Beyotime, China) and quantified using a BCA kit (Beyotime, China). An equivalent amount of protein extracts was loaded onto 10\% SDS-PAGE and then transferred onto PVDF membranes. The membranes were blocked in 5\% non-fat milk in TBST at room temperature for $2 \mathrm{~h}$ and then incubated with primary antibodies overnight at $4{ }^{\circ} \mathrm{C}$. The membranes were then washed and incubated with a secondary antibody. After washing, the blots were developed with a ECL system (Pierce, Rockford, IL, USA). Primary antibodies against p21 and $\beta$-actin were obtained from (Cell Signaling Technology, USA).

\section{Cell proliferation assay and cloning formation assay}

The Cell Counting Kit-8 (CCK-8) method was utilised to detect cell proliferation. Transfected cells were seeded into 96 -well plates at a density of 2000 cells per well in triplicate. Cell viability was measured by the CCK-8 system (Dojindo, Japan) at 0, 24, 48, 72 and $96 \mathrm{~h}$ after seeding in accordance with the manufacturer's instructions.

For colony formation assay, transfected cells were seeded into six-well plates at a density of 200 cells per well and maintained in DMEM medium containing 10\% FBS for 2 weeks. The colonies were imaged and counted after they were fixed and stained.

\section{Migration and invasion assays}

At $24 \mathrm{~h}$ after transfection, $2 \times 10^{4}$ cells were suspended with $200 \mu \mathrm{L}$ of serum-free medium and then seeded into the upper chambers ( $8 \mathrm{~mm}$ pore size, Costar). Medium containing 10\% FBS was added to the bottom chamber as a chemoattractant. The upper chamber was coated with or without BD Matrigel (BD Biosciences, USA) for the invasion or migration assays. The cells were incubated at $37^{\circ} \mathrm{C}$ for $48 \mathrm{~h}$ for the invasion assay or $24 \mathrm{~h}$ for the migration assay. Subsequently, cells at the top chamber were removed with cotton swabs, and those on the lower surface were fixed with methanol, stained with $0.1 \%$ crystal violet and then photographed under a microscope at $100 \times$ magnification (Olympus, Japan).

\section{FACS analysis}

To detect cell cycle, transfected cells were stained with propidium iodide utilising the Cycletest Plus DNA Reagent Kit (BD Biosciences) in accordance with the manufacturer's instructions. The ratios of cells in the G0/G1 phase to the S phase were compared with controls.

\section{RNA binding protein immunoprecipitation assay}

RNA binding protein immunoprecipitation (RIP) assay was performed using the Magna RIP Kit (Millipore, USA) and Ago2 antibody (Cell Signaling Technology, USA) in accordance with the manufacturer's instructions. In brief, $10^{7}$ transfected cells were washed in ice-cold PBS twice, lysed in an equal volume of RIP lysis buffer and then incubated with $5 \mu$ g of primary antibodies for $2 \mathrm{~h}$ at $4{ }^{\circ} \mathrm{C}$. Subsequently, $50 \mu \mathrm{L}$ 


\section{Cellular Physiology Cell Physiol Biochem 2018;46:1606-1616 \begin{tabular}{l|l} 
DOI: 10.1159/000489208 & $\begin{array}{l}\text { O 2018 The Author(s). Published by S. Karger AG, Basel } \\
\text { wwww.karger.com/cpb }\end{array}$
\end{tabular} \\ Li et al.: CircRNA-Cdrlas Inhibits Bladder Cancer Proliferation by Sponging microRNA- \\ $135 a$}

of prepared magnetic beads was added to each sample and incubated at $4{ }^{\circ} \mathrm{C}$ overnight. The beads were washed briefly with RIP buffer for five times and resuspended in $500 \mu \mathrm{L}$ of TRIzol LS (Life Technology, USA). The binging products were detected by qRT-PCR.

\section{RNA antisense purification}

The specific biotinylated probes that hybridise to Cdr1as were synthesised by (Gongsi, China). The sequences were as follows: Cdr1as sense: 5'-GGT GCC ATC GGA AAC CCT GGA TAT TGC AGA CA-3'-Biotin and Cdr1as antisense: 5'-TGT CTG CAA TAT CCA GGG TTT CCG ATG GCA CC-3'-Biotin. The experiment was performed as previously described [24]. In brief, $10^{7}$ cells were washed with PBS and lysed in $500 \mu \mathrm{L}$ of lysis buffer. The Cdr1as-specific biotinylated sense and anti-sense probes were added to the circRNA-RNA antisense purification (RAP) system and incubated for $2 \mathrm{~h}$ at room temperature. Subsequently, $50 \mu \mathrm{L}$ of blocked streptavidin magnetic beads (Invitrogen) was added to each reaction tube and incubated for $4 \mathrm{~h}$ at $4{ }^{\circ} \mathrm{C}$. The beads were then washed briefly with lysis buffer for five times. TRIzol LS (Life Technology, USA) was used to recover miRNAs specifically interacting with Cdr1as.

\section{Biotin pull down assay}

This experiment was performed as previously described [25]. Cells were transfected with $50 \mu \mathrm{M}$ of biotinylated miRNA mimic or nonsense control (NC) and lysed in $500 \mu \mathrm{L}$ of lysis buffer. Subsequently, $50 \mu \mathrm{L}$ of blocked streptavidin magnetic beads (Invitrogen) was added to each reaction tube and incubated for 4 $\mathrm{h}$ at $4{ }^{\circ} \mathrm{C}$. The beads were then washed briefly with lysis buffer for five times and resuspended in $500 \mu \mathrm{L}$ of TRIzol LS (Life Technology, USA) to recover ncRNAs specifically interacting with miRNAs.

\section{RNA FISH}

To detect the location of Cdr1as and miR-135a, we used DNA oligo probes (GenePharma, Shanghai, China) labelled with Cy5 for Cdr1as and FAM for miRNAs. The sequences were as follows: Cdr1as: $5^{\prime}$-GGT GCC ATC GGA AAC CCT GGA TAT TGC AGA CA-3'-Cy5 and miR-135a-5p: 5'-TCA CAT AGG AAT AAA AAG CCA TA-3'-Fam. H293T cells were grown to the exponential phase and were $80 \%-95 \%$ confluent at the time of fixation. After prehybridisation (1 PBS/0.5\% Triton X-100), the cells were hybridised in hybridisation buffer ( $40 \%$ formamide, $10 \%$ dextran sulfate, $1 \times$ Denhardt's solution, $4 \times \mathrm{SSC}, 10 \mathrm{mM} \mathrm{DDT}, 1 \mathrm{mg} \mathrm{mL}^{-1}$ yeast transfer RNA, and $1 \mathrm{mg} \mathrm{mL}^{-1}$ sheared salmon sperm DNA) with Cy5-labelled probes specific to Cdr1as and Famlabelled probes specific to miR135a at $42{ }^{\circ} \mathrm{C}$ overnight. Nuclei were counterstained with 4 , 6-diamidino2-phenylindole. The images were acquired using a Leica SP5 confocal microscope (Leica Microsystems, Mannheim, Germany).

\section{Northern blot}

The specific biotinylated probes that hybridise to Cdr1as and 18s RNA were synthesised by (Gongsi, China). The sequences were as follows: Cdr1as: 5'-GGT GCC ATC GGA AAC CCT GGA TAT TGC AGA CA-3'Biotin and 18S RNA: 5'-CGG AAC TAC GAC GGT ATC TG-3'-Biotin. Total RNAs were extracted with TRIzol LS reagent (Life Technology, USA). The experiments were performed using Northern blot kit (Ambion, USA) in accordance with the manufacturer's instructions. In brief, $30 \mu \mathrm{g}$ of total RNAs was mixed with three volumes of Formaldehyde Load Dye and electrophoresed in a 1\% agarose-formaldehyde gel. The RNAs were then transferred onto a Hybond-N+ nylon membrane (Beyotime, China) and hybridised with biotin-labelled DNA probes overnight. The bands were developed with a Biotin Chromogenic Detection Kit (Thermo Scientific, USA) in accordance with the manufacturer's instructions.

\section{Animal studies}

The animal studies were approved by the Animal Management Committee of Nanjing Medical University. Female athymic Balb/c nude mice ( 4 weeks old, $n=10$ ) were used. About $10^{7}$ transfected T24 cells and control cells were injected subcutaneously into the axilla of mice. The width (W) and length (L) of the tumours were measured using calipers every 3 days, and the volume (V) was calculated using the formula $\mathrm{V}=(\mathrm{W} 2 \times \mathrm{L}) / 2$. At 4 weeks after injection, the mice were sacrificed, and tumour weights were measured. The tumours were fixed in $4 \%$ formalin for immunohistochemical analysis.

\section{Statistical analysis}

Data were analysed using SPSS version 19.0 and presented as means \pm standard deviation. Student's t-test was performed to analyse differences between the two groups, and $p<0.05$ was considered to indicate 


\section{Cellular Physiology Cell Physiol Biochem 2018;46:1606-1616 \begin{tabular}{l|l} 
DOI: 10.1159/000489208 & and Biochemistry 2018 The Author(s). Published by S. Karger AG, Basel \\
wublished & anarger.com/cpb
\end{tabular} Li et al.: CircRNA-Cdrlas Inhibits Bladder Cancer Proliferation by Sponging microRNA- $135 a$}

Fig. 1. Cdr1as is downregulated in bladder cancer specimens. A: Relative expression level of Cdr1as in bladder cancer tissues and adjacent normal tissues(ANTs)(n=94), Cdr1as was significantly downregulated in bladder cancer tissues than ANTs; B: The transfection efficiency of Cdr1as overexpression adenovirus is detected by fluorescence microscope,(Left: excited with bluish light; Right: excited with white light); C: The expression level of Cdr1as in adenovirus transfected cells and Circ-controltransfected cells; D: Northern blot of Cdr1as in cells transfected with Cdr1as or Circ-control adenovirus.

statistical significance. All experiments were repeated more than three times, and each experiment was performed in triplicate.

\section{Results}

Expression of CircRNA-Cdr1as is down-regulated in bladder cancer specimens

To explore the expression levels of Cdr1as, qRT-PCR was performed in 94 pairs of bladder cancer tissues and ANTs. Results suggested that Cdr1as was significantly down-regulated in bladder cancer tissues compared with ANTs ( $p<0.05$, Fig. $1 \mathrm{~A})$. This result suggests that Cdr1as serves as a tumour suppressor factor in bladder cancer.

Overexpression of Cdr1as inhibits the proliferation, invasion and migration of bladder cancer cells

To investigate the functions of Cdr1as in bladder cancer, the Cdr1as overexpression adenovirus was constructed and transfected into EJ, T24, 253J and RT4 cell lines. Fluorescence micrographs showed 90\% transfection efficiency (Fig. 1B). The up-regulation of Cdr1as in bladder cancer cells was confirmed by qRT-PCR (Fig. 1C). For further study, EJ and T24 cells were selected as representatives.
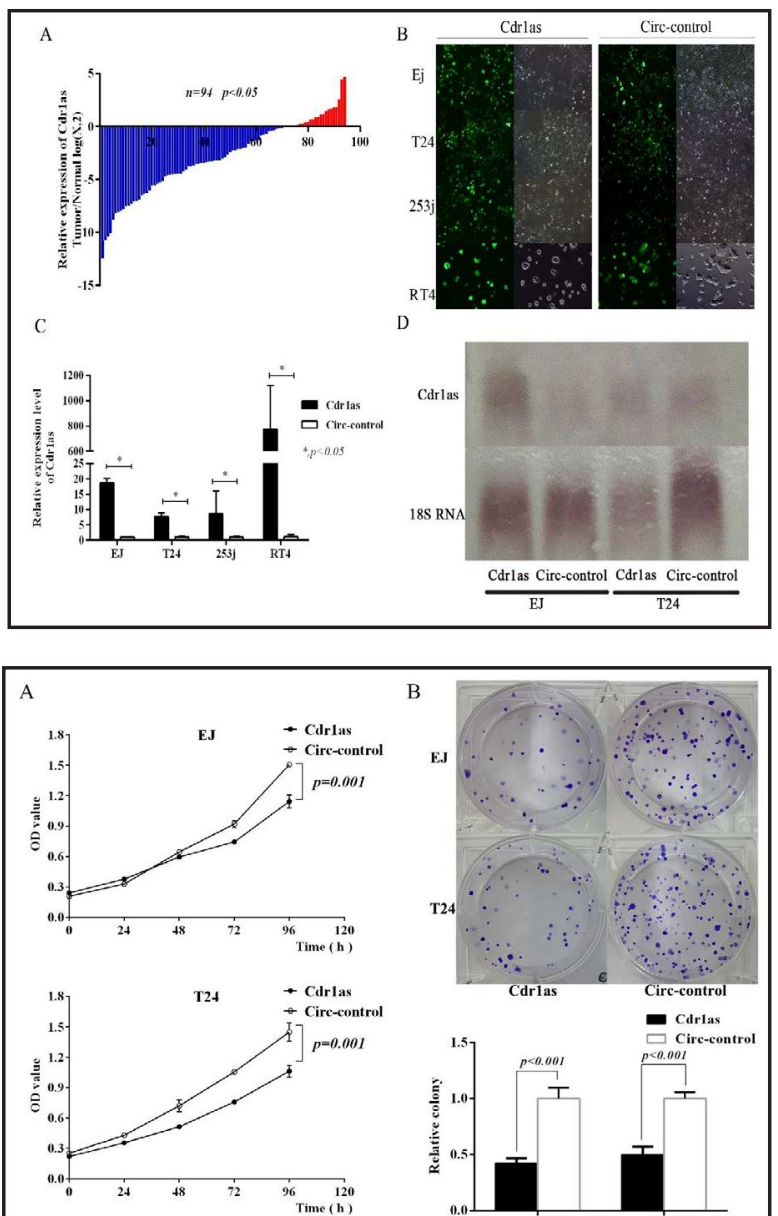

C
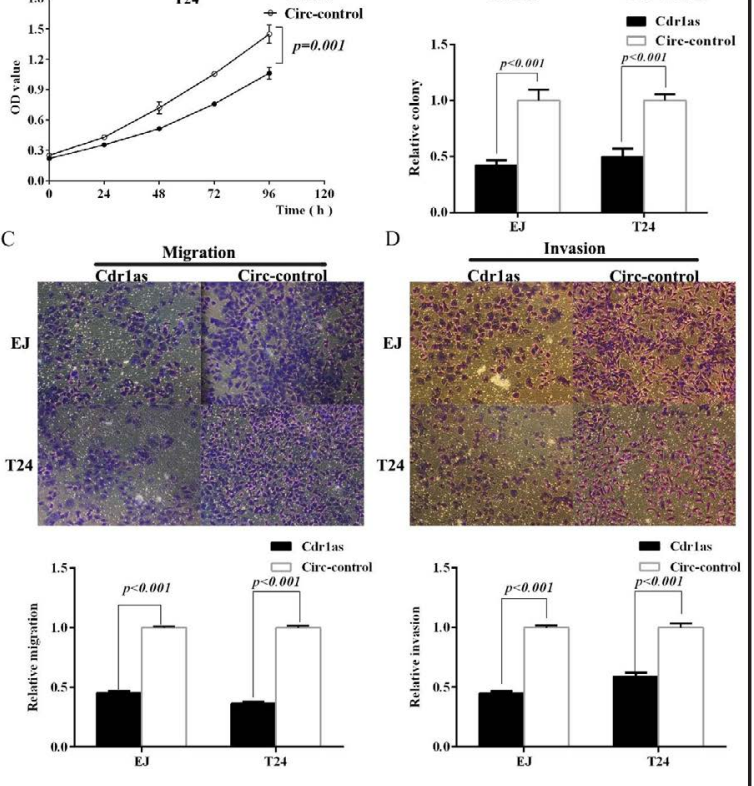

Fig. 2. Cdr1as inhibits the proliferation, invasion and migration of bladder cancer cell A\&B: Cell viability was inhibited in Cdr1as overexpression cells using the CCK-8 method and cloning formation assay; C\&D: Representative images of the migration and invasion assays, cell migration and invasion were inhibited in Cdr1as overexpression cells. Northern blot assay indicated that Cdr1as was significantly up-regulated at the RNA level in the cells transfected with the Cdr1as adenovirus (Fig. 1D). 


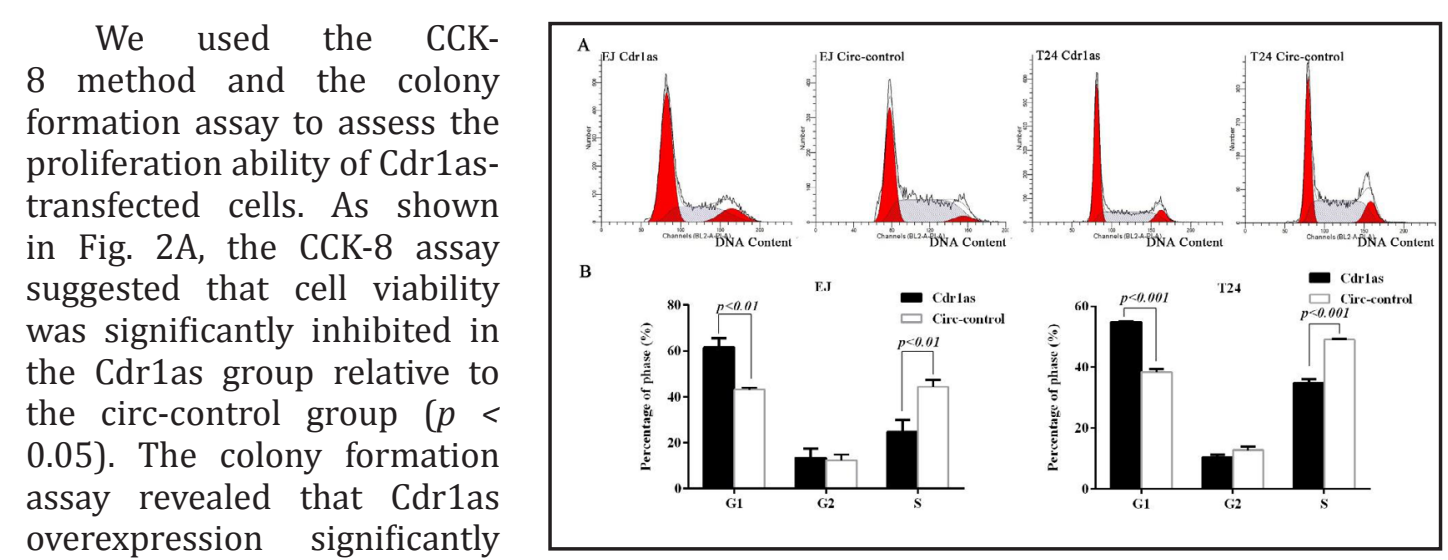
reduced the formation of clones ( $p=0.001$, Fig. 2B). Furthermore, transwell migration and invasion assays were performed, and the results indicated that Cdr1as can suppress the migration and invasion capabilities of EJ and T24 cells (Fig. 2C and 2D).

Cdr1as represses cell cycle entry in bladder cancer cells

Flow cytometry was performed to detect the effect of Cdr1as on the cell cycle. Compared with Gfp, Cdr1as significantly increased the number of cells distributed in the G1 phase and decreased the number of cells in the $\mathrm{S}$ phase $(p<$ 0.05 , Fig. 3). These results suggest that Cdr1as induces cell cycle arrest in bladder cancer cells.
Fig. 3. Cdr1as represses cell cycle entry in bladder cancer cells. A: Cell cycle distribution of cells transfected with Cdr1as or Circcontrol adenovirus. B: Cdr1as induced cell cycle arrest in G1 phase.

Overexpression of Cdr1as suppresses the growth of tumour xenografts in nude mice

To evaluate the effect of Cdr1as on tumour growth in vivo, we injected the transfected T24 cells subcutaneously into nude mice. Tumour volumes were assessed weekly after implantation. As shown in Fig. 4, Cdr1as up-regulation significantly suppressed the growth of tumour xenografts in nude mice. In addition, tumour volume was significantly reduced in the Cdr1as group relative to the circ-control group ( $p=0.005$, Fig. 4C). Four weeks later, all mice were sacrificed, and the weights of tumours were measured. Average weight was obviously lighter in the Cdr1as-transfected cells than in the circ-control group ( $p<0.05$, Fig. 4B). The results above were consistent with those in vitro.

\section{Cdr1as serves as a sponge for multiple miRNAs in bladder cancer}

We utilised starBase V2.0 (http://starbase.sysu.edu.cn/), CircInteractome (http:// circinteractome.nia.nih.gov/), CircNet (http://circnet.mbc.nctu.edu.tw) and miRDB (http:// mirdb.org/miRDB/index.html) to find potential miRNA target sites on Cdr1as. As shown in Fig. 5A, different miRNAs were predicted using different programs. For further study, we performed Ago2 RIP assay. The results of qRT-PCR showed that Cdr1as pulled down by Ago 2 was 10 -fold more than that by IgG in the Cdr1as-transfected cells ( $p=0.002$, Fig. 5B). Four miRNAs(miR-1290, miR-1246, miR-7 and miR-135a) pulled down by Ago2 in Cdr1as- 
Fig. 5. Cdr1as serves as a sponge for multiple miRNAs in bladder cancer. A: Potential miRNA target sites on Cdr1as were predicted utilizing the four programs; B: Ago2 RNA immunoprecipitation (RIP) assay for the amount of Cdr1as inT24 cell overexpressing Cdr1as or Circ-control; C: Relative expression level of the four miRNAs immunoprecipitated by Ago2 in Cdr1as overexpressing or control cells; D: Relative expression level of the four miRNAs in Cdr1as overexpression cells immunoprecipitated by Ago2 or IgG; E: Relative expression level of Cdr1as pull-down by biotinylated probes than anti-sense probes in Cdr1as overexpressing or control cells; F : Relative expression level of the four miRNAs pull-down by biotinylated probes than antisense probes in Cdr1as overexpression cells.

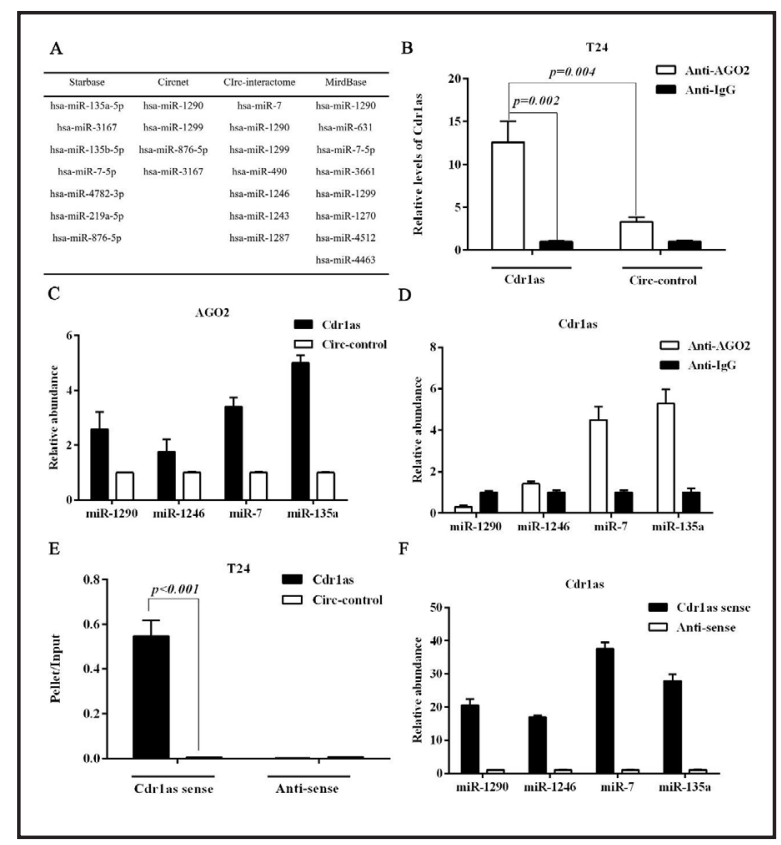

Fig. 6. Cdr1as sponges miR-7/ miR-135a and inhibits the activity of miR-135a in bladder cancer. A\&B: qRT-PCR analysis of Cdr1as level in the streptavidin captured fractions from cell lysates after transfection with biotinylated miR7 and miR-135a than control RNA (NC). C\&D: Relative expression level of miR-7 and miR-135a in bladder cancer tissues and $\operatorname{ANTs}(n=29) ; \quad$ E\&F: Correlation analysis of the expression level of Cdr1as and miR-7 or miR-135a; G: Proliferation assessed using a CCK8 kit T24 cells transfected with four miRNA mimics or control RNA; $\mathrm{H}$ : Co-localization between Cdr1as and miR-135a was observed by FISH, Nuclei were stained with DAPI; I\&J: P21 protein in miR-135a
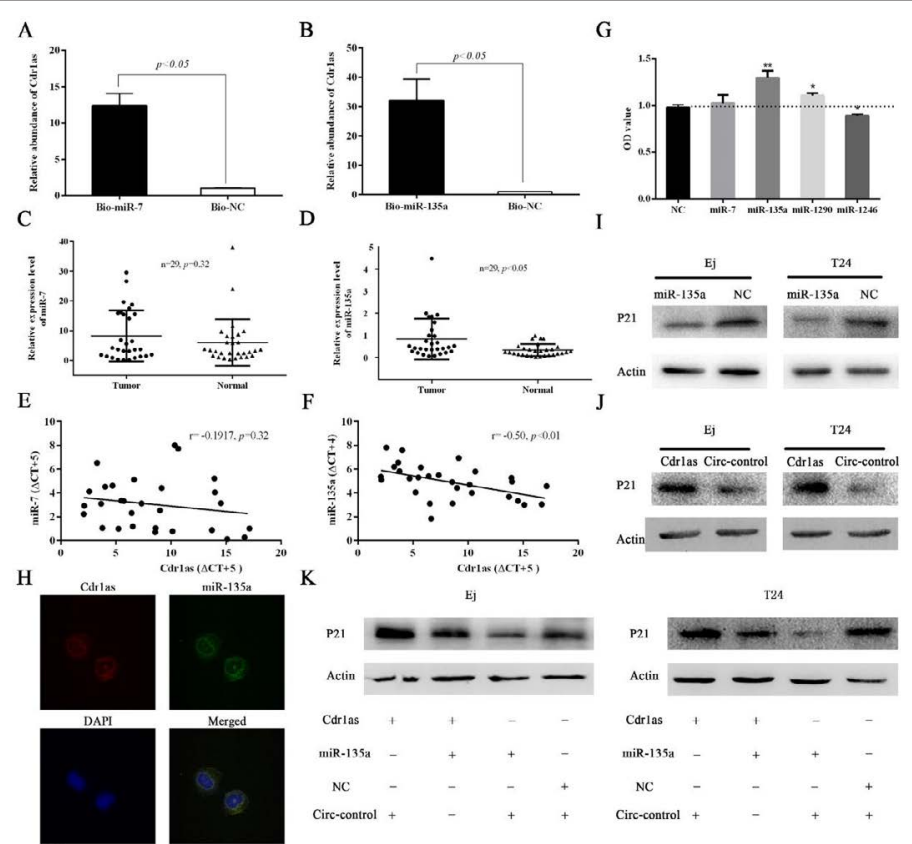

$\mathrm{H}$
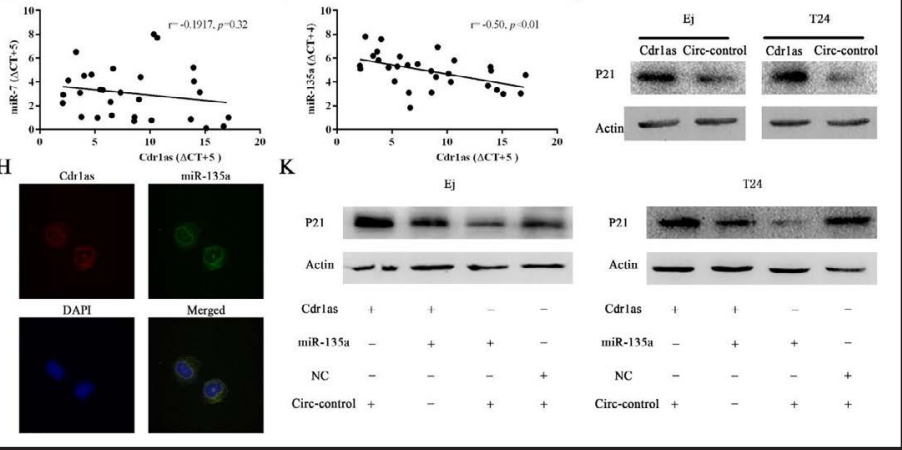

or NC and Cdr1as or Circ-control

transfected cells. K: Western blot analysis showed that Cdr1as partly rescued the miR-135a-induced p21 downregulation in EJ and T24 cells.

transfected cells were about 2-fold more than that in Circ-control transfected cells (Fig. 3C). Furthermore, three miRNAs (miR-7, miR-135a and miR-1246) showed higher expression when pulled down by Ago2 than IgG in Cdr1as-transfected cells(Fig. 3D). Other miRNAs showed no significant differences (data not shown). The results suggest that Cdr1as can serve as a platform for the interaction between miRNAs and Ago2.

RAP assay was utilised to explore the relationship between Cdr1as and miRNAs. The biotinylated probes (Cdr1as sense) pulled down much more Cdr1as than the anti-sense probes in Cdr1as overexpression cells ( $p<0.001$, Fig. 5E), indicating that the probes can 


\section{Cellular Physiology Cell Physiol Biochem 2018;46:1606-1616

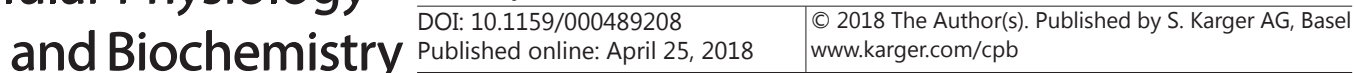 \\ Li et al.: CircRNA-Cdr1as Inhibits Bladder Cancer Proliferation by Sponging microRNA- \\ $135 \mathrm{a}$}

bind to Cdr1as specifically. Moreover, the miRNAs pulled down by Cdr1as sense probes were about 20-40-fold more than those by Anti-sense probes in Cdr1as-overexpressing cells (Fig. $5 \mathrm{~F})$. These results indicate that Cdr1as can serve as a sponge for multiple miRNAs in bladder cancer.

Cdr1as sponges miR-7/miR-135a and inhibits the activity of miR-135a in bladder cancer

As shown in Fig. 5C and 5F, much more miR-7 and miR-135a were pulled down by Cdr1as. Therefore, we chose miR-7 and miR-135a for further study. In the biotin pull-down assay, more Cdr1as was detected by qRT-PCR in the Bio-miR-7- and Bio-miR-135a-transfected cells than in the Bio-NC-transfected cells (Fig. 6A and 6B), suggesting that miR-7 and miR-135a can bind to and pull down Cdr1as. For further study, 29 pairs of bladder cancer tissues and ANTs were used to detect the expression of miR-7 and miR-135a. Results suggested that miR-135a was up-regulated in bladder cancer tissues (Fig. 6D), but similar results were not observed for miR-7 (Fig. 6C). Furthermore, correlation analysis demonstrated that the expression of Cdr1as showed a moderate negative correlation with miR-135a $(\mathrm{r}=-0.50, p$ $<0.01$, Fig. 6F). However, no correlation was found between Cdr1as and miR-7 in bladder cancer ( $\mathrm{r}=-0.1917, p=0.32$, Fig. $6 \mathrm{E}$ ).

In addition, we transfected individual miRNA mimics into T24 cells. The most striking pro-proliferative effect was observed in the miR-135a-transfected cells (Fig. 6G). However, miR-7 showed no biological functions in bladder cancer cells (data not shown). These results suggest that Cdr1as exerts anti-oncogenic functions via sponging miR-135a but not miR-7 in bladder cancer. The results of the co-localisation experiment was consistent with the interaction of Cdr1as and miR-135a (Fig. $6 \mathrm{H}$ ). A previous study reported that miR135 a promotes the proliferation and cell cycle progression of bladder cancer and inhibits the expression of p21 in bladder cancer [26]. The result was validated in the miR-135atransfected EJ and T24 cells (Fig. 6I). Moreover, the expression of p21 was up-regulated in the Cdr1as-transfected cells (Fig. 6J), indicating the interaction of Cdr1as with miR-135a. To confirm that Cdr1as exerts anti-oncogenic functions via sponging miR-135a, we performed a restoration experiment by transfecting Cdr1as and miR-135a into bladder cancer cells simultaneously. Results showed that Cdr1as rescued the inhibitory effect of miR-135a on p21 (Fig. 6K). Overall, these results indicate that Cdr1as sponges miR-7/miR-135a and inhibits the activity of miR-135a in bladder cancer.

\section{Discussion}

Recently, a large amount of circRNAs have been identified by high-throughput deep RNA sequencing coupled with powerful computational analyses [27-29]. However, little is known about the production, transportation and functions of circRNAs. Several studies suggest that circRNAs regulate gene expression and participate in numerous cellular processes by sponging miRNAs. For example, Zheng et al. found that circHIPK3 can regulate cell growth by sponging multiple miRNAs [12]. Du et al. reported that Foxo3 circular RNA retards cell cycle progression by forming ternary complexes with p21 and CDK2 [16].

In the field of tumours, circRNAs have also been reported to be functional. Yang et al. revealed that silencing of cZNF292 circular RNA can suppress human glioma tube formation via the Wnt/beta-catenin signalling pathway [14]. Similarly, cir-ITCH inhibits the proliferation of ESCC by sponging miR-7, miR-17 and miR-214. To date, only two studies [30, 31] have revealed the altered expression of circRNAs in bladder cancer, indicating that circRNAs play important roles in the development and progression of bladder cancer.

A previous study reported that Cdr1as acts as an oncogene in hepatocellular carcinoma [23]. However, we found that Cdr1as was down-regulated in bladder cancer tissues compared with ANTs. This contradictory result aroused our interest to explore further the function of Cdr1as in bladder cancer. Subsequently, functional experiments were performed. The results suggested that the up-regulation of Cdr1as can inhibit the proliferation, migration and 


\section{Cellular Physiology Cell Physiol Biochem 2018;46:1606-1616

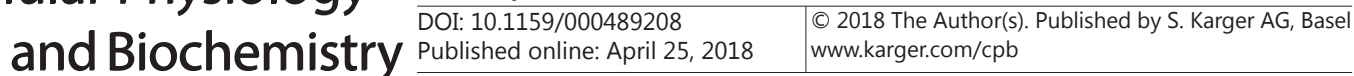 \\ Li et al.: CircRNA-Cdr1as Inhibits Bladder Cancer Proliferation by Sponging microRNA- \\ $135 a$}

invasion of bladder cancer cells. Furthermore, an in vivo animal study indicated that Cdr1as can slow down tumour formation. Our results were in contrast with those of a previous study [23], in which Cdr1as was up-regulated in hepatocellular carcinoma while down-regulated in bladder cancer.

We found that Cdr1as can sponge multiple miRNAs, including miR-7, miR-135a and miR-1290, in bladder cancer. The Ago2 RIP and RAP assays showed that much more miRNAs, especially miR-7 and miR-135a, were pulled down by Cdr1as compared with oligo probes. Biotin pull-down assay revealed that more Cdr1as was detected by qRT-PCR in the BiomiR-7- or Bio-miR-135a-transfected cells than in the Bio-NC-transfected cells, suggesting that miR-7 and miR-135a can bind to and pull down Cdr1as. These results indicate that Cdr1as serves as a molecular sponge for multiple miRNAs in bladder cancer.

A previous study has reported that Cdr1as serves as a molecular sponge of miR-7 and conducts important functions in different biological or pathological progresses, including myocardial infarction [32], hepatocellular carcinoma [23], insulin transcription and secretion [22] and brain development [22]. We detected the expression of miR-7 and miR-135a in 29 pairs of bladder cancer tissues and ANTs. We hypothesised that Cdr1as also exerts its functions by sponging miR-7 in bladder cancer. However, the results suggested that miR135a was up-regulated in bladder cancer tissues, and the expression of miR-7 did not show significant difference between bladder cancer tissues and ANTs. In addition, correlation analysis showed a moderate negative correlation between the expression of Cdr1as and miR-135a but not miR-7. Moreover, proliferation assay showed a striking pro-proliferative effect in the miR-135a-transfected cells other than the miR-7-transfected cells (data not shown). Therefore, we conjectured that Cdr1as exerts anti-oncogenic functions via sponging miR-135a in bladder cancer. The following co-localisation experiment further strengthened the evidence of the interaction between Cdr1as and miR-135a.

MiR-135a reportedly enhances cellular proliferation in multiple malignancies, including colorectal cancer [33], hepatocellular carcinoma [34], and melanoma [35]. It also purportedly promotes tumour formation in bladder cancer and suppresses p21 expression [26]. We detected the expression level of p21 in miR-135a- or Cdr1as-transfected cells. The results showed that miR-135a can down-regulate the expression of p21, whereas an opposite effect was observed for Cdr1as. Furthermore, a rescue experiment suggested that Cdr1as can partly reverse the inhibitory effect of miR-135a on p21, indicating an interaction between Cdr1as and miR-135a. Cdr1as conducts its functions partly via p21 up-regulation by sponging miR135a. However, the underlying mechanism needs further investigation.

Basing on the present findings, we conclude that Cdr1as sponges multiple miRNAs in bladder cancer and exerts anti-oncogenic functions by sponging microRNA-135a. The CircRNA-miRNA-target gene regulatory network plays important roles in bladder cancer. Targeting this novel pathway may provide a potential idea in the diagnosis and treatment of bladder cancer.

\section{Acknowledgements}

This work was supported by the National Natural Science Foundation of China (grants No. 81772711 and 81602235), the Priority Academic Program Development of Jiangsu Higher Education Institutions (PAPD), Jiangsu Provincial Special Program of Medical Science (BL2012027), the Provincial Initiative Program for Excellency Disciplines of Jiangsu Province (grant no. BE2016791), the "333" project of Jiangsu Province (LGY2016002), Six talent peaks project of Jiangsu Province(2015-wsw-033) and Jiangsu Province's Key Provincial Talents Program (ZDRCA2016006). The funders had no role in study design, data collection and analysis, decision to publish, or preparation of the manuscript. The authors also want to thank Dr. T. Hansen. (Aarhus University, Denmark) for providing Cdr1as overexpression plasmid as described in Materials and Methods. 


\section{Cellular Physiology Cell Physiol Biochem 2018;46:1606-1616 \begin{tabular}{l|l} 
and Biochemistry Published online: April 25, 2018 & $\begin{array}{l}\text { (c) } 2018 \text { The Author(s). Published by S. Karger AG, Basel } \\
\text { www.karger.com/cpb }\end{array}$
\end{tabular} \\ Li et al.: CircRNA-Cdrlas Inhibits Bladder Cancer Proliferation by Sponging microRNA- $135 \mathrm{a}$}

\section{Disclosure Statement}

Neither author has a competing interest to disclose.

\section{References}

1 Antoni S, Ferlay J, Soerjomataram I, Znaor A, Jemal A, Bray F: Bladder Cancer Incidence and Mortality: A Global Overview and Recent Trends. Eur Urol 2016;10.1016/j.eururo.2016.06.010

-2 Ooms EC, Anderson WA, Alons CL, Boon ME, Veldhuizen RW: Analysis of the performance of pathologists in the grading of bladder tumors. Hum Pathol 1983;14:140-143.

-3 Pop-Bica C, Gulei D, Cojocneanu-Petric R, Braicu C, Petrut B, Berindan-Neagoe I: Understanding the Role of Non-Coding RNAs in Bladder Cancer: From Dark Matter to Valuable Therapeutic Targets. Int J Mol Sci 2017;18:

4 Shang A, Yang M, Shen F, Wang J, Wei J, Wang W, Lu W, Wang C, Wang C: MiR-1-3p Suppresses the Proliferation, Invasion and Migration of Bladder Cancer Cells by Up-Regulating SFRP1 Expression. Cell Physiol Biochem 2017;41:1179-1188.

5 Zhi Y, Pan J, Shen W, He P, Zheng J, Zhou X, Lu G, Chen Z, Zhou Z: Ginkgolide B Inhibits Human Bladder Cancer Cell Migration and Invasion Through MicroRNA-223-3p. Cell Physiol Biochem 2016;39:1787-1794.

-6 Li P, Yang X, Cheng Y, Zhang X, Yang C, Deng X, Li P, Tao J, Yang H, Wei J, Tang J, Yuan W, Lu Q Xu X, Gu M: MicroRNA-218 Increases the Sensitivity of Bladder Cancer to Cisplatin by Targeting Glut1. Cell Physiol Biochem 2017;41:921-932.

7 Xiong Y, Wang L, Li Y, Chen M, He W, Qi L: The Long Non-Coding RNA XIST Interacted with MiR-124 to Modulate Bladder Cancer Growth, Invasion and Migration by Targeting Androgen Receptor (AR). Cell Physiol Biochem 2017;43:405-418.

-8 Zhu H, Li X, Song Y, Zhang P, Xiao Y, Xing Y: Long non-coding RNA ANRIL is up-regulated in bladder cancer and regulates bladder cancer cell proliferation and apoptosis through the intrinsic pathway. Biochem Biophys Res Commun 2015;467:223-228.

-9 Fan Y, Shen B, Tan M, Mu X, Qin Y, Zhang F, Liu Y: TGF-beta-induced upregulation of malat1 promotes bladder cancer metastasis by associating with suz12. Clin Cancer Res 2014;20:1531-1541.

10 Chen LL, Yang L: Regulation of circRNA biogenesis. RNA Biol 2015;12:381-388.

11 Memczak S, Jens M, Elefsinioti A, Torti F, Krueger J, Rybak A, Maier L, Mackowiak SD, Gregersen LH, Munschauer M, Loewer A, Ziebold U, Landthaler M, Kocks C, le Noble F, Rajewsky N: Circular RNAs are a large class of animal RNAs with regulatory potency. Nature 2013;495:333-338.

12 Zheng Q, Bao C, Guo W, Li S, Chen J, Chen B, Luo Y, Lyu D, Li Y, Shi G, Liang L, Gu J, He X, Huang S: Circular RNA profiling reveals an abundant circHIPK3 that regulates cell growth by sponging multiple miRNAs. Nat Commun 2016;7:11215.

-13 Zhao ZJ, Shen J: Circular RNA participates in the carcinogenesis and the malignant behavior of cancer. RNA Biol 2015;10.1080/15476286.2015.11221621-8.

14 Yang P, Qiu Z, Jiang Y, Dong L, Yang W, Gu C, Li G, Zhu Y: Silencing of cZNF292 circular RNA suppresses human glioma tube formation via the Wnt/beta-catenin signaling pathway. Oncotarget 2016;10.18632/ oncotarget.11523

15 Xie H, Ren X, Xin S, Lan X, Lu G, Lin Y, Yang S, Zeng Z, Liao W, Ding YQ Liang L: Emerging roles of circRNA_001569 targeting miR-145 in the proliferation and invasion of colorectal cancer. Oncotarget 2016;7:26680-26691.

16 Du WW, Yang W, Liu E, Yang Z, Dhaliwal P, Yang BB: Foxo3 circular RNA retards cell cycle progression via forming ternary complexes with p21 and CDK2. Nucleic Acids Res 2016;44:2846-2858.

17 Guarnerio J, Bezzi M, Jeong JC, Paffenholz SV, Berry K, Naldini MM, Lo-Coco F, Tay Y, Beck AH, Pandolfi PP: Oncogenic Role of Fusion-circRNAs Derived from Cancer-Associated Chromosomal Translocations. Cell 2016;165:289-302.

18 Xu L, Zhang M, Zheng X, Yi P, Lan C, Xu M: The circular RNA ciRS-7 (Cdr1as) acts as a risk factor of hepatic microvascular invasion in hepatocellular carcinoma. J Cancer Res Clin Oncol 2016;10.1161/ circresaha.116.309568 


\section{Cellular Physiology Cell Physiol Biochem 2018;46:1606-1616 and Biochemistry Published online: April 25, $2018 \quad \begin{aligned} & \text { DOI 10159/2018 The Author(s). Published by S. Karger AG, Basel } \\ & \text { www.karger.com/cpb }\end{aligned}$ \\ Li et al.: CircRNA-Cdr1as Inhibits Bladder Cancer Proliferation by Sponging microRNA- \\ $135 a$}

19 Bachmayr-Heyda A, Reiner AT, Auer K, Sukhbaatar N, Aust S, Bachleitner-Hofmann T, Mesteri I, Grunt TW, Zeillinger R, Pils D: Correlation of circular RNA abundance with proliferation--exemplified with colorectal and ovarian cancer, idiopathic lung fibrosis, and normal human tissues. Sci Rep 2015;5:8057.

20 Li P, Chen S, Chen H, Mo X, Li T, Shao Y, Xiao B, Guo J: Using circular RNA as a novel type of biomarker in the screening of gastric cancer. Clin Chim Acta 2015;444:132-136.

21 Hansen TB, Kjems J, Damgaard CK: Circular RNA and miR-7 in cancer. Cancer Res 2013;73:5609-5612.

22 Xu H, Guo S, Li W, Yu P: The circular RNA Cdr1as, via miR-7 and its targets, regulates insulin transcription and secretion in islet cells. Sci Rep 2015;5:12453.

-23 Yu L, Gong X, Sun L, Zhou Q, Lu B, Zhu L: The Circular RNA Cdr1as Act as an Oncogene in Hepatocellular Carcinoma through Targeting miR-7 Expression. PLoS One 2016;11:e0158347.

24 Nan A, Chen L, Zhang N, Liu Z, Yang T, Wang Z, Yang C, Jiang Y: A novel regulatory network among LncRpa, CircRar1, MiR-671 and apoptotic genes promotes lead-induced neuronal cell apoptosis. Arch Toxicol 2016;10.1007/s00204-016-1837-1

25 Tan SM, Lieberman J: Capture and Identification of miRNA Targets by Biotin Pulldown and RNA-seq. Methods Mol Biol 2016;1358:211-228.

26 Mao XP, Zhang LS, Huang B, Zhou SY, Liao J, Chen LW, Qiu SP, Chen JX: Mir-135a enhances cellular proliferation through post-transcriptionally regulating PHLPP2 and FOXO1 in human bladder cancer. J Transl Med 2015;13:86.

-27 Starke S, Jost I, Rossbach 0, Schneider T, Schreiner S, Hung LH, Bindereif A: Exon circularization requires canonical splice signals. Cell Rep 2015;10:103-111.

-28 Sand M, Bechara FG, Sand D, Gambichler T, Hahn SA, Bromba M, Stockfleth E, Hessam S: Circular RNA expression in basal cell carcinoma. Epigenomics 2016;8:619-632.

-29 Sand M, Bechara FG, Gambichler T, Sand D, Bromba M, Hahn SA, Stockfleth E, Hessam S: Circular RNA expression in cutaneous squamous cell carcinoma. J Dermatol Sci 2016;83:210-218.

-30 Huang M, Zhong Z, Lv M, Shu J, Tian Q, Chen J: Comprehensive analysis of differentially expressed profiles of IncRNAs and circRNAs with associated co-expression and ceRNA networks in bladder carcinoma. Oncotarget 2016;10.18632/oncotarget.9706

-31 Zhong Z, Lv M, Chen J: Screening differential circular RNA expression profiles reveals the regulatory role of circTCF25-miR-103a-3p/miR-107-CDK6 pathway in bladder carcinoma. Sci Rep 2016;6:30919.

-32 Geng HH, Li R, Su YM, Xiao J, Pan M, Cai XX, Ji XP: The Circular RNA Cdr1as Promotes Myocardial Infarction by Mediating the Regulation of miR-7a on Its Target Genes Expression. PLoS One 2016;11:e0151753.

33 Zhou W, Li X, Liu F, Xiao Z, He M, Shen S, Liu S: MiR-135a promotes growth and invasion of colorectal cancer via metastasis suppressor 1 in vitro. Acta Biochim Biophys Sin (Shanghai) 2012;44:838-846.

-34 Zeng YB, Liang XH, Zhang GX, Jiang N, Zhang T, Huang JY, Zhang L, Zeng XC: miRNA-135a promotes hepatocellular carcinoma cell migration and invasion by targeting forkhead box 01. Cancer Cell Int 2016;16:63.

-35 Ren JW, Li ZJ, Tu C: MiR-135 post-transcriptionally regulates FOX01 expression and promotes cell proliferation in human malignant melanoma cells. Int J Clin Exp Pathol 2015;8:6356-6366. 